\title{
LAS COMUNIDADES DE QUIRONÓMIDOS (DIPTERA: CHIRONOMIDAE) DEL RÍO ULLA (NW DE ESPAÑA)
}

\author{
F. Cobo y M.A. González
}

Departamento de Biología Animal, Facultad de Biología, Universidad de Santiago de Compostela. Spain.

Palabras clave: Chironomidae, drift, distribution, regulated river, Galicia, Spain.

\begin{abstract}
CHIRONOMID MIDGE (DIPTERA: CHIRONOMIDAE) COMMUNITIES FROM THE RIVER ULLA (NW SPAIN)

Using drift nets, pupal exuviae of Chironomidae were caught in the river Ulla during 1986. Regulation by impoundment markedly altered the chironomidean fauna. Species richness and drift density were typically reduced at the regulated sampling station. Taxonomical and ecological classification, cluster analysis, and quantitative parameters were used to elucidate patterns of community structure from survey data. Data of emergence periods of main systematic groups in given.
\end{abstract}

\section{INTRODUCCIÓN}

En trabajos anteriores, nos hemos ocupado de la distribución, ecología y períodos de emergencia de las principales especies de quironómidos del río Ulla (Сово, 1988; Сово et al., en prensa). En la presente publicación expondremos los resultados obtenidos del estudio de la estructura y composición de sus comunidades. Para la realización de este trabajo se ha contado con fondos del proyecto PB 86 - 0406 de la CICYT.

\section{MATERIAL Y MÉTODOS}

Tal y como explicamos en detalle en el trabajo Сово $\boldsymbol{e t}$ al. (en prensa), se han establecido siete estaciones de muestreo a lo largo del curso del río Ulla: 1 Monterroso (500 m, UTM: 29TNH9338), 2 Santiso (320 m, UTM: 29TNH7944), 3 Hortas

Limnetica, 6: 109-118 (1990)

(C) Asociación Española de Limnología, Madrid, Spain
(100 m, UTM: 29TNH6544), 4 Puente Ledesma (80 m, UTM: 29TNH5438), 5 Puente Sarandón (40 m, UTM: 29TNH4533), 6 Puentevea (30 m, UTM: 29TNH3734) y 7 Puentecesures (20 m, UTM: 29TNH2830).

Hemos muestreado en cada estación mensualmente durante el año 1986, utilizando una red de deriva tipo Brundin de $250 \mu \mathrm{m}$ de luz de malla, $33 \mathrm{~cm}$ de diámetro y $60 \mathrm{~cm}$ de longitud para la captura de pupas y exuvias pupales. Esta técnica resulta ventajosa con respecto a la recogida tradicional de imagos o larvas, puesto que además de su facilidad de ejecución, permite integrar en una misma muestra diversos microbiotopos y proporciona un número de individuos suficientemente representativo de la composición específica y estructura de la comunidad. Sin embargo, la interpretación de los resultados ha de tener en cuenta algunos problemas inherentes a la propia metodología, como la representatividad en el espacio y en el tiempo o el propio tamaño de la muestra. El número de exuvias capturadas, y por tanto el número de especies presentes en las muestras, de- 
pende de la densidad de deriva y de la cantidad de agua filtrada, que depende a su vez de la velocidad de la corriente y del tiempo de exposición de las redes. Con el fin de simplificar el análisis de los resultados, hemos transformado nuestros datos de captura en términos de densidad de deriva (n. ${ }^{\circ}$ de individuos $1100 \mathrm{~m}^{3}$ de agua filtrada), si bien en todos los casos hemos filtrado en torno a los $600 \mathrm{~m}^{3}$ de agua, después de exponer las redes entre una y dos horas, dependiendo de la velocidad de la corriente.

Simultáneamente a la captura de la fauna se ha evaluado una serie de parámetros fisicoquímicos (Cово et al., opus cit.) de cuyo análisis se pueden extraer algunas generalidades que afectan a toda la masa de agua estudiada.

\section{RESULTADOS}

\section{Características fisicoquímicas}

La temperatura mínima del agua registrada en el curso del Ulla durante el período de muestreo fue de $6,6^{\circ} \mathrm{C}$ en diciembre en la estacion 1 y la máxima de $21^{\circ} \mathrm{C}$ en julio en la estacion 2 . La temperatura aumenta hacia la desembocadura de forma más o menos uniforme, existiendo una discontinuidad en la estación 3 por efecto de las descargas del embalse de Portodemouros situado aguas arriba. Las diferencias térmicas más acusadas tienen lugar en la estación 2 (máxima, $21^{\circ} \mathrm{C}$ el 30/VII/86, y mínima, $7,3^{\circ} \mathrm{C}$ el $28 / \mathrm{XII} / 86$ ) y las menos acentuadaqs en la estación 4 (máxima, $18,6^{\circ} \mathrm{C}$ el $30 / \mathrm{VII} / 86$, y mínima, $8,6^{\circ} \mathrm{C}$ el 3011186 ).

Los valores de la concentración de $\mathrm{O}_{2}$ se encuentran por encima de los 7,5 mg/l (83\% de saturación). Se trata, pues, de valores totalmente normales para las aguas corrientes, cuyo valor inferior según KLEIN (1959) ha de superar el $75 \%$ de saturación.

La conductividad de las aguas del Ulla es baja a lo largo de su curso (máxima, $220 \mu \mathrm{S} / \mathrm{cm}$ en la estación 7, y mínima, 40,4 $\mu \mathrm{S} / \mathrm{cm}$ en la estación 1), correspondiendo, sus valores medios, con los valores de las clases 2 (muy débil) y 3 (débil) de Nisbet \& Verneaux (1970), propias de cursos de agua oligotróficos, con sustratos ácidos. Sin embargo, puntualmente pueden observarse valores moderados en la estación 4 (máxima, 200 $\mu \mathrm{S} / \mathrm{cm}$ ), como consecuencia de los aportes del río Brandelos, que recibe las aguas ácidas de drenaje de una mina de cobre situada en sus inmediaciones, y en la estación 7 por su proximidad al mar.

La evolución longitudinal del pH revela la existencia de un mínimo en la estación 4 (media, 6,6), mientras que en las demás estaciones está muy próximo a la neutralidad (entre 6,9 y 7,1).

El contenido en nitratos y nitritos $(1,2 \mathrm{mg} / \mathrm{l} \mathrm{y}$ 5,6 $\mu \mathrm{g} / \mathrm{l}$ de media respectivamente), así como la ausencia de amonio en todo el curso del río, denota un buen estado de la capacidad de autodepuración de las aguas del Ulla; solamente entradas muy puntuales de materia orgánica elevan los valores de estos parámetros, sin que superen en ningún momento a los normales en un río de estas características (máximos: $3,5 \mathrm{mg} / \mathrm{l}$ y $31,7 \mu \mathrm{g} / \mathrm{l}$ ).

Los máximos de la alcalinidad total se manifiestan en las estaciones $2\left(25,5 \mathrm{mg}\right.$ de $\left.\mathrm{CO}_{3} \mathrm{Ca} / \mathrm{l}\right)$ y 3 (25,4 mg de $\left.\mathrm{CO}_{3} \mathrm{Ca} / \mathrm{l}\right)$.

Tanto la dureza y el contenido en $\mathrm{Ca}^{+2} \mathrm{y} \mathrm{Mg}^{+2}$ como el contenido en sulfatos, presentan máximos apreciables en la estación 4 (10,2 mg/l de $\mathrm{Ca}^{+2}, 6,8$ $\mathrm{mg} / \mathrm{l}$ de $\mathrm{Mg}^{+2}$ y $70,2 \mathrm{mg} / \mathrm{l}$ de $\mathrm{SO}_{4}^{-2}$ ), lo que revela la moderada mineralización existente en ese tramo de río.

La variación longitudinal de los ortofosfatos es muy irregular. Es muy probable que los valores máximos $(48,0 \mu \mathrm{g}$ de $\mathrm{P} / \mathrm{l}$ en la estación 1 y 45,0 $\mu \mathrm{g}$ de P/l en la estación 7) se correspondan con las entradas puntuales de detergentes al río, como consecuencia de su proximidad a zonas urbanas.

Los cloruros permanecen más o menos constantes y dentro de los límites normales a lo largo del río (media, $12,6 \mathrm{mg} / \mathrm{l}$ ), con la excepción de la estación 7 (máximo, 29,7 mgll), en la que juega un papel determinante la influencia de las mareas.

La materia orgánica disuelta (absorbancia a 275 nm) disminuye en el tramo medio del río, en correspondencia con lo observado en la evolución de los nitritos y nitratos.

Con la intención de obtener una imagen de las similitudes existentes entre las estaciones desde el punto de vista fisicoquímico, hemos aplicado el coeficiente de correlación de PEARson (1977) a los valores medios de cada parámetro, transformándolos previamente en logaritmos, y las hemos agrupado en dendrogramas (fig. 1) mediante el método jerárquico del mínimo, máximo y media ponderada (UPGMA) de SoKal \& Michener 


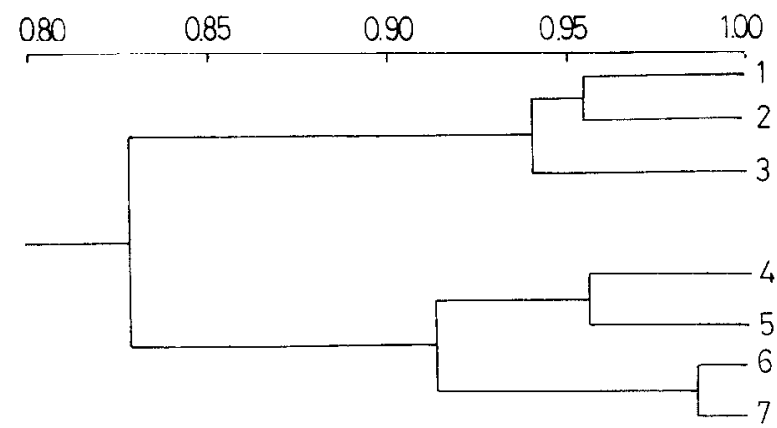

Figura 1.- Dendrograma de similitud entre estaciones a partir de los datos fisicoquímicos, según el coeficiente de correlación de Pearson (1977).

Similarity cluster analysis of sampling points based on physico-chemical data by Pearson's correlation coefficient.

(1958), de forma que aparecen tres grupos de estaciones claramente definidos: en primer lugar, el formado por las dos estaciones de cabecera, en segundo lugar el formado únicamente por la estación 3 y en tercero el grupo constituido por las cuatro estaciones inferiores, siendo estos agrupamientos muy similares a los obtenidos con los datos relativos a la fauna.

\section{Fenología y ecología}

Antes de abordar el estudio de la fenología de las especies en las cuatro estaciones del año, quisimos comprobar si éstas se correspondían con los patrones generales de emergencia de las especies en virtud de la afinidad faunística entre los meses. Los resultados obtenidos se resumen en el dendrograma de afinidad obtenido (fig. 2) después de la utilización del coeficiente de Sorensen (1948) y según el método UPGMA de Sokal \& Michener (opus cit.).

Existen cuatro períodos del año que engloban meses cuya fauna es cualitativamente similar. El primer grupo, que podíamos equiparar al invierno, abarca los meses de noviembre hasta marzo; los meses de abril y mayo conforman el segundo grupo, que correspondería a la primavera; un tercer grupo, formado por los meses de junio, julio y agosto, constituye el período de verano; y finalmente los meses de otoño, septiembre y octubre, aparecen, de igual forma que los de primavera, como una etapa de transición entre los dos grandes grupos perfectamente individualizados por su composición faunística, el correspondiente al invierno y el correspondiente al verano.

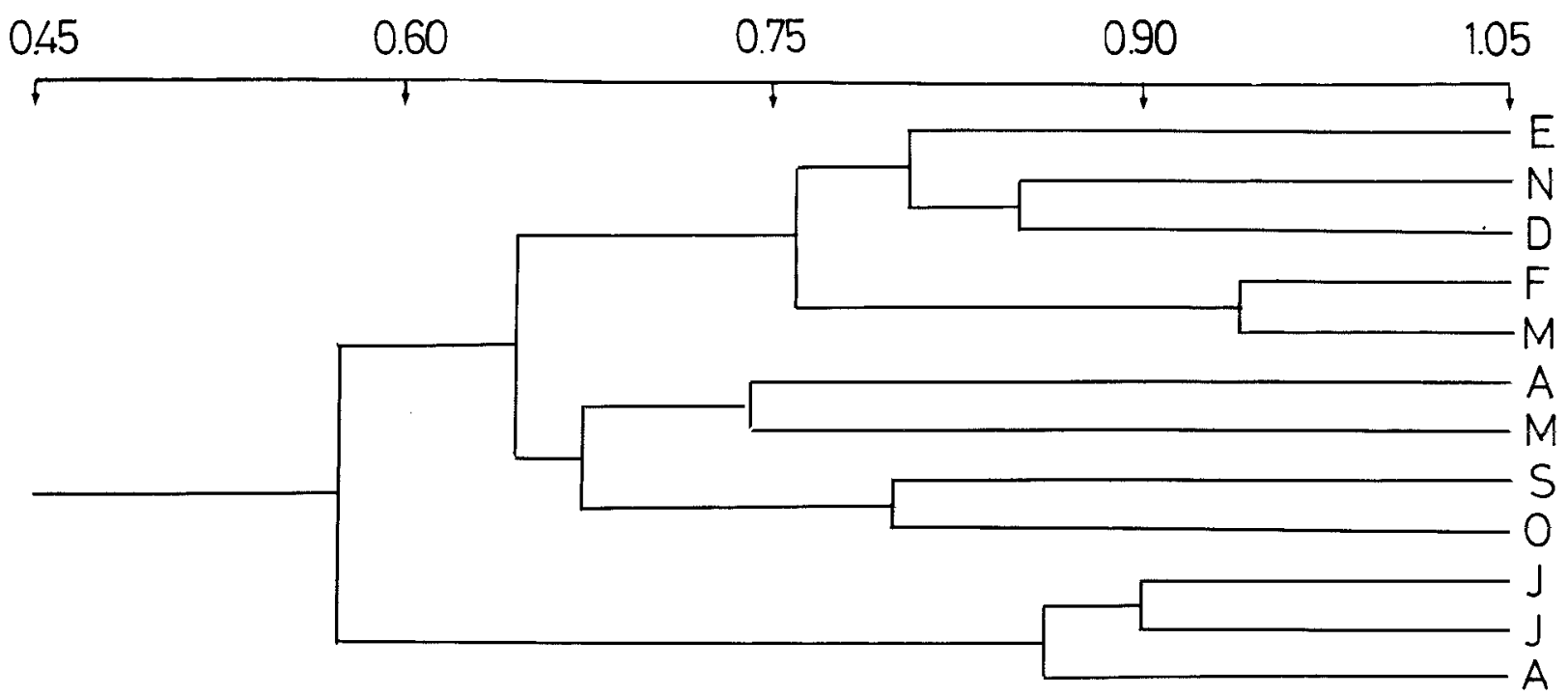

Figura 2.- Dendrogramas de similitud entre los meses, según sus afinidades faunísticas. Clustering of months by faunistical affinities. 


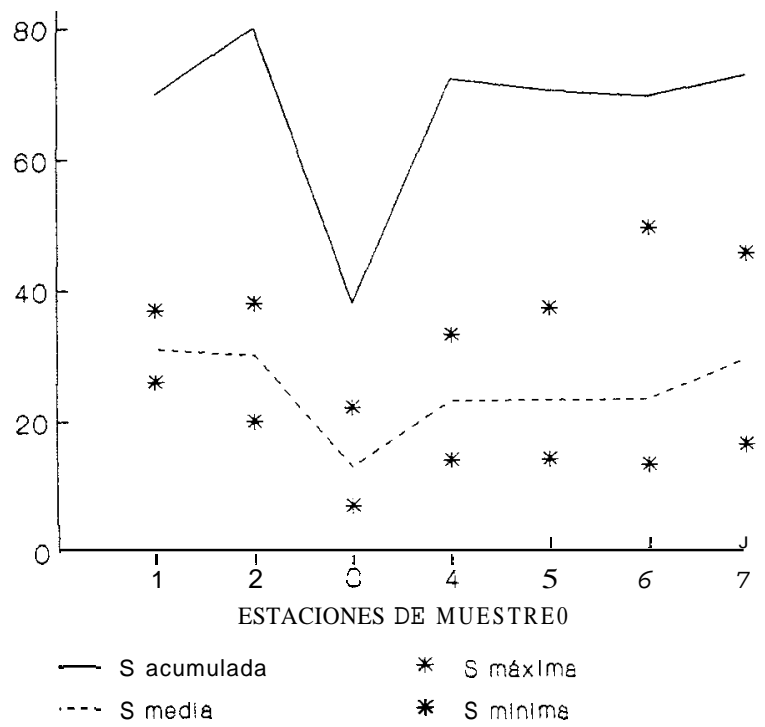

Figura 3.- Variación longitudinal de la riqueza específica. Longitudinal vanation of species richness.

Esta forma de distribuirse los meses del año en función de la presencia o ausencia de las especies, coincide en términos generales con las características climáticas que presenta cada uno de ellos en la cuenca estudiada (Rodríguez, 1982), lo que corrobora en cierta medida la hipótesis de que los períodos de emergencia de los quironómidos están sensiblemente influenciados por las particularidades climáticas de la zona.

a) Riqueza específica y densidad de deriva

El número total de especies capturadas en el no Ulla a lo largo del año asciende a 117. La gráfica de la variación espacial de la riqueza específica (fig. 3) muestra un acusado descenso en la estación 3 , en la que apenas si se hallan representadas el $32 \%$ de las especies inventariadas, hecho que contrasta fuertemente con lo observado en la estación precedente, con relación a la cual se aprecia una pérdida del $52,5 \%$ y donde se ha obtenido la mayor riqueza específica (80 especies). Dicha figura revela valores más bajos en las estaciones situadas aguas abajo del embalse que en las situadas aguas arriba. Es interesante señalar, también, que el coeficiente de variabilidad aplicado $(V=\sigma / \bar{s} 100)$ señala a la estación 2 como la más estable en cuanto a su riqueza específica, mientras que de nuevo la estación 3 refleja la mayor inestabilidad.

En la tabla 1 presentamos, para cada estación, el número de especies y las frecuencias relativas de los principales grupos (subfamilias y tribus) correspondientes al conjunto de las capturas anuales. Los Orthocladiinae (63 especies, 53,8 \%) son los más abundantes en el conjunto del río, principalmente en la estación de cabecera, seguidos ya a una cierta distancia de los Chironomini (24 especies, 20,5\%); los Tanytarsini y Tanypodinae están representados por 15 y 10 especies respectivamente, y la incidencia de los restantes grupos es meramente testimonial.

Los Orthocladiinae dominan igualmente todas las estaciones, puesto que superan en todas ellas el $50 \%$ de la comunidad y alcanzan sus cotas máximas (57 especies, $\mathbf{7 2 , 9} \%$ ) en la cabecera (estación 1) y en la estación 3, donde se registra el menor número de especies de este grupo (28 especies), si bien su representación porcentual es la más elevada $(73,6 \%)$. Los Chironomini están muy poco diversificados en la estación de cabecera (2 especies, 2,8 \%). En todas las demás representan entre un $9,7 \%$ (estación 3 ) y un $17,3 \%$ (estación 6). Los Tanytarsini y Tanypodinae mantienen una incidencia regular a lo largo del curso del Ulla, salvo en la estación 3, donde experimentan una importante merma en consonancia con lo observado también para los restantes grupos. Los Diamesini están pobremente representados (de 1 a 3 especies, según las estaciones). La única especie de Prodiamesinae, Prodiamesa olivacea, aparece en las estaciones 4 y 7, y Buchonomyia thiennemani, presente únicamente en la estación 2 , constituye asimismo el único representante de la subfamilia Buchonomyiinae.

El estudio de la evolución anual de la riqueza específica por campaña de muestreo (fig. 3) revela que alcanza su valor máximo en primavera, durante la cual se han capturado 99 especies $(84,6 \%)$. A partir del verano asistimos a un progresivo descenso, que alcanza el mínimo durante el invierno, en el que se capturaron 64 especies $(54,7 \%)$. Los meses de mayo (75 especies, $64,1 \%$ ) y diciembre (43 especies, $36,7 \%$ ) regis- 
Tabla 1.- Número de especies y abundancia relativa de los principales grupos taxonómicos en cada estación de muestreo. Number of species and relative abundance of main taxonomical groups in each sampling stations.

\begin{tabular}{|c|c|c|c|c|c|c|c|c|}
\hline \multirow[b]{3}{*}{ Grupo } & \multicolumn{7}{|c|}{ Estaciones de muestreo } & \multirow{3}{*}{ Total } \\
\hline & \multicolumn{2}{|r|}{2} & 3 & 4 & 5 & 6 & 7 & \\
\hline & spp. $\%$ & spp. $\%$ & spp. $\%$ & spp. $\%$ & spp. $\%$ & spp. $\%$ & spp. $\%$ & \\
\hline Tanypodinae & $5-7,1$ & $7-8,7$ & $1-2,6$ & $5-6,9$ & $5-7,1$ & $5-7,2$ & $6-8,3$ & $10-8,5$ \\
\hline Buchonomyiinae & - & $1-1,2$ & - & - & - & - & - & $1-0,8$ \\
\hline Diamesinae & $1-1,4$ & $1-1,2$ & $1-2,6$ & $1-1,3$ & $1-1,4$ & $2-2,8$ & $3-4,1$ & $3-2,5$ \\
\hline Prodiamesinae & - & - & - & $1-1,3$ & - & - & $1-1,3$ & $1-0,8$ \\
\hline Orthocladiinae & $57-72,9$ & $47-58,7$ & $28-73,6$ & $43-59,7$ & $44-62,8$ & $40-57,9$ & $44-61,1$ & $63-53,8$ \\
\hline Chironomini & $2-2,8$ & $13-16,2$ & $5-13,1$ & $12-16,6$ & $9-12,8$ & $12-17,3$ & $7-9,7$ & $24-20,5$ \\
\hline Tanytarsini & $11-15,7$ & $11-13,7$ & $3-7,8$ & $10-13,8$ & $11-15,7$ & $10-14,4$ & $11-15,2$ & $15-12,8$ \\
\hline Total & 70 & 80 & 38 & 72 & 70 & 69 & 72 & 117 \\
\hline
\end{tabular}

tran los valores extremos al respecto. La riqueza específica máxima por campaña de muestreo se ha registrado en el mes de mayo, con 45 especies en la estación 2, y la riqueza específica mínima por campaña de muestreo se sitúa en el mes de enero, en la estación 3 y con 6 especies. El mes más

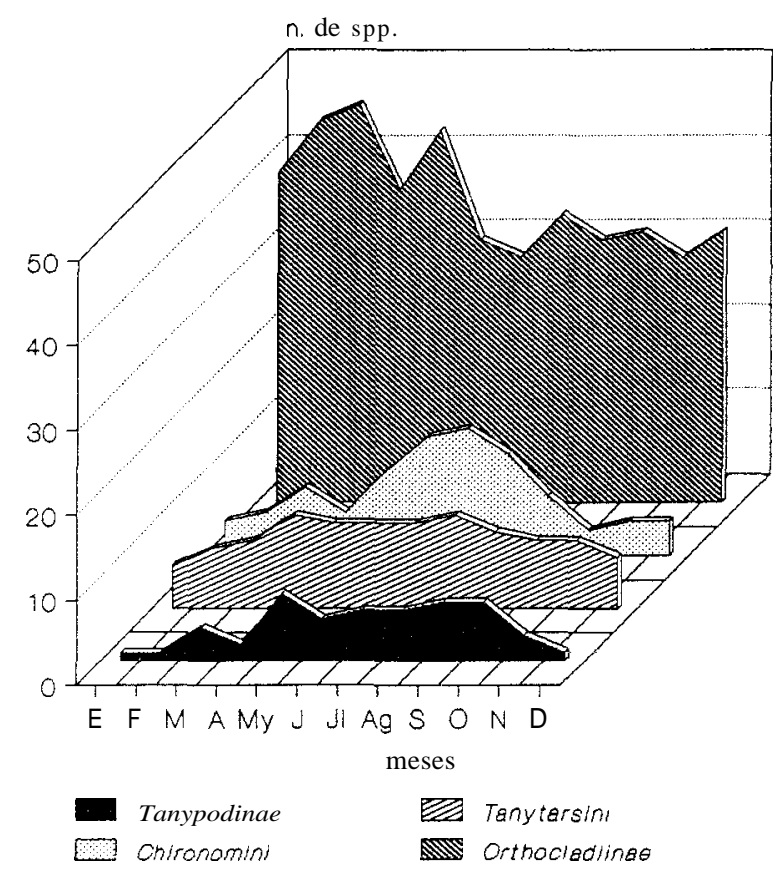

Figura 4.- Variación mensual de la riqueza específica de los principales grupos taxonómicos.

Monthly variation of species richness of main taxonomical groups. uniforme en cuanto al número de especies capturadas en cada estación fue septiembre, mientras que en enero y mayo se han observado las mayores variaciones.

Las variaciones anuales de la riqueza específica de los diferentes grupos se refleja en la figura 4. Especialmente llamativo resulta el hecho de que los Orthocladiinae estén presentes en su práctica totalidad $(90,4 \%)$ durante la primavera, mientras que durante el verano y otoño existe una importante merma de sus efectivos $(60,3$ y $66,6 \%$, respectivamente) y el invierno supone un período de recuperación, en el que su riqueza específica $(79,3 \%)$ tiende ya a aproximarse al máximo primaveral. Los Chironomini alcanzan su mayor riqueza durante el verano (75\%) y primavera $(66,6 \%)$, pero en invierno $\rightarrow$ diferencia de lo observado con los Orthocladiinae - ésta desciende a sus niveles más bajos $(20,8 \%)$. En los Tanytarsini el análisis de la evolución mensual revela un paulatino ascenso desde el mínimo, que corresponde al mes de enero (5 especies), hasta el mes de abril (11 especies), manteniéndose constante a partir de entonces hasta el final del verano, donde se inicia un progresivo descenso hasta el invierno. Finalmente, los Tanypodinae experimentan una drástica reducción durante el invierno, mientras que en el resto del año su riqueza específica mantiene una constancia aceptable.

En el ciclo anual, los valores de la densidad de deriva (fig. 5) ascienden progresivamente desde enero hasta el máximo del mes de julio, para posteriormente descender de forma ligeramente más 
rápida hasta los valores mínimos registrados durante el invierno.

b) Afinidad entre estaciones

Se han agrupado las estaciones según sus afinidades faunísticas. Uno de los coeficientes utilizados, el de Sorensen (1948), de uso generalizado, se basa únicamente en el criterio de presencia-ausencia, y aunque su interés parezca relativamente limitado, nos permiten, sin embargo, integrar la información recogida en varias series de muestreos, dado que no reflejan más que su aspecto cualitativo. Las comparaciones cuantitativas se han efectuado mediante la utilización del coeficiente de RaAbe (1952), que tiene en cuenta las abundancias relativas de las diferentes especies.

Las matrices de asociación obtenidas entre las distintas estaciones, en base a los coeficientes utilizados, han permitido agruparlas de forma jerárquica mediante el método del mínimo, máximo y media ponderada (UPGMA) de Sokal \& Michener (1958).

La configuración de los dendrogramas obtenidos (fig. 6) refleja el estado de un ecosistema perturbado, en el que las diferentes estaciones no se

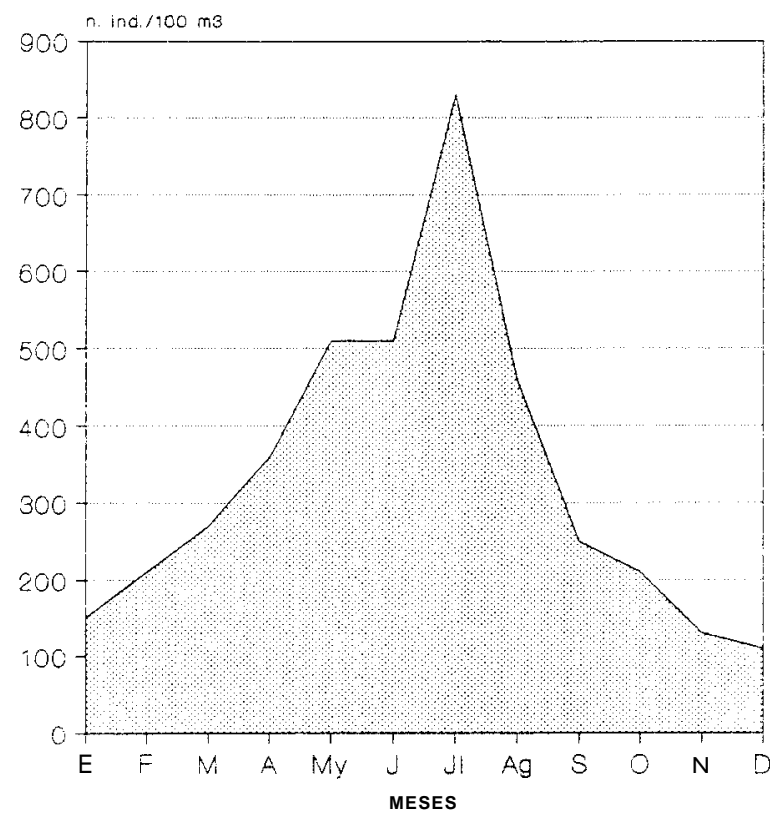

Figura 5.- Variación mensual de la densidad de deriva. Monthly variation of drift density.
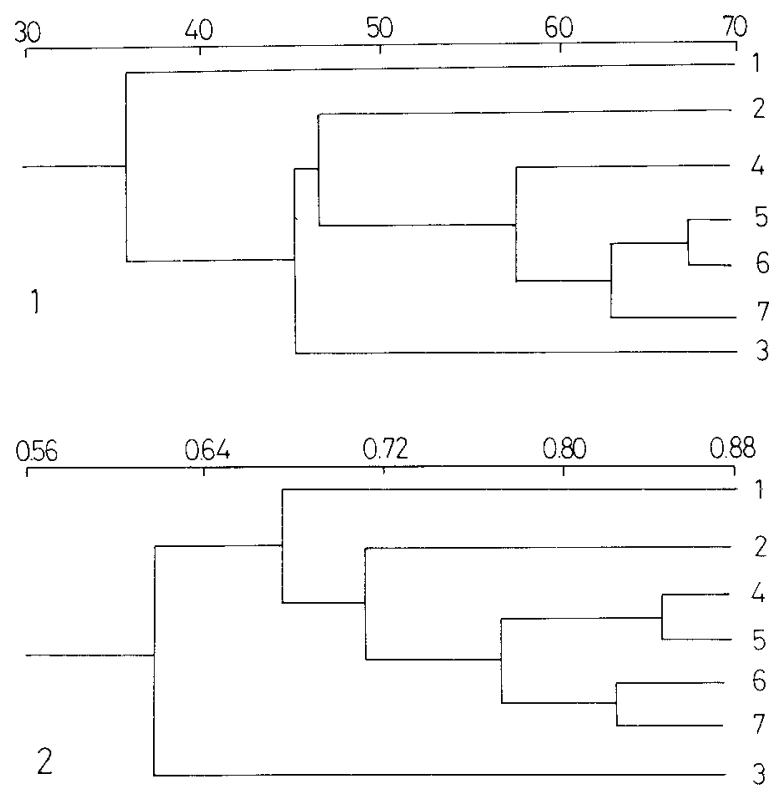

Figura 6.- Dendrogramas de similitud faunística entre estaciones: 1 de Raabe, 2 de Sorensen.

Cluster analysis of sampling estations by faunistical similarity; 1 from Raabe, 2 from Sorensen.

ordenan de forma contínua y los valores de los coeficientes son poco elevados, incluso entre estaciones geográficamente próximas. Valorando en conjunto los métodos de análisis, podemos distinguir cuatro grupos: los tres primeros están constituidos por estaciones aisladas $(1,2,3)$ y el cuarto, sin embargo, agrupa las cuatro estaciones inferiores, caracterizadas por una similar composición y abundancia relativa de los principales taxones.

De los cuatro grupos mencionados, el mejor individualizado por los coeficientes utilizados es el formado por la estación 1, el cual presenta la mínima afinidad según Raabe, con relación al resto de las estaciones. Está situado en la cota altitudinal más elevada de las estaciones muestreadas $(500 \mathrm{~m})$. Se caracteriza por su alta tasa de Orthocladiinae (57 spp., $72,9 \%$ ) y por la escasa representación de Chironomini. Las tres especies más frecuentes en el conjunto de las capturas son: Corynoneura lobata, Corynoneura edwardsi, y Thienemanniella acuticornis, especies típicamente reófilas y estenotermas de aguas frías, si exceptuamos C. lobata, que se muestra según nuestros datos como una especie euritopa. Once especies 
aparecen principalmente en esta estación con relación a las demás, de las cuales las ocho primeras son exclusivas: Nilotanypus dubius, Zavrelimyia barbatipes, Thienemannimyia Pe 1, Orthocladius frigidus, Tvetenia bavarica, Psectrocladius psilopterus, Phaenopsectra camptolabis, Micropsectra notescens, Polypedilum uncinatum, Rheotanytarsus distinctissimus y Stempellinella brevis.

La siguiente estación mejor individualizada es la 2. En ella continúa registrándose una elevada tasa de Orthocladiinae (47 spp., 58,9\%), pero a diferencia de la anterior, los Chironomini aumentan hasta alcanzar las $13 \mathrm{spp}$. (16,2\%). Rheocricotopus chalybeatus, Orthocladius (s. str.) oblidens y Potthastia gaedii son especies frecuentes en la estación, si bien sólo $O$. (s. str.) oblidens aparece durante todo el año, mientras que $R$. chalybeatus está ausente en los meses de invierno y $P$. gaedii sólo aparece durante el verano, pero con un gran número de individuos. Ocho especies son más abundantes en esta estación que en las demás: Buchonomyia thienemanni, Eurycnemus crassipes, Dicrotendipes ssp., Microtendipes sp., Paracladopelma nigritula, Polypedilum nubeculosum, Xenochironomus xenolabis y Paratanytarsus confusus. Las siete primeras son exclusivas (4 Chironomini, 1 Orthocladiinae, 1 Buchonomyinae y 1 Tanytarsini) y la mayor parte de ellas están presentes en los meses de junio, julio y agosto, época en la que mejor se individualizala estación, que se caracteriza además por un abundante desarrollo de macrófitas. Las diferencias establecidas entre la estación 1 y 2 probablemente han de atribuirse más a la posición de las estaciones en el río, que a una posible perturbación, dadas las particularidades faunísticas de cada una de ellas y la similitud existente con relación a la fisicoquímica de sus aguas.

El grupo III (estación 3) es el mejor segregado en el dendrograma del coeficiente cualitativo. La influencia que sobre él tiene la actividad del embalse, que origina cambios anárquicos en el caudal, se traduce en una merma importante de la riqueza específica y en el número de los efectivos de aquellas especies que son abundantes en las demás estaciones. Este estado de imprevisibilidad sólo es tolerado por algunas especies. El gran número de ejemplares de Orthocladius rhyacobius, capturados en abril, hace que esta especie se manifieste como abundante en el total anual de la es- tación; además $\boldsymbol{O}$. oblidens y $\boldsymbol{O}$. saxicola son muy abundantes e incluso dominantes durante varios meses del año. Esta estación se caracteriza por la presencia de Eukiefferiella lobifera y Stictochironomus maculipennis como especies exclusivas, además de por la abundancia de las tres especies anteriormente señaladas.

En el grupo IV (estaciones 4, 5, 6 y 7) existe una representatividad comparable entre las subfamilias Orthocladiinae y Chironominae. Las cuatro estaciones de muestreo comparten un elevado número de especies comunes, con abundancias similares que dan homogeneidad al grupo. Entre ellas, las más frecuentes son: las especies eurioicas del género Orthocladius, como $O$. oblidens y $\boldsymbol{O}$. saxicola, Cricotopus bicinctus, Paratrichocladius rufiventris, Corynoneura lobata, Rheocricotopus chalybeatus, Virgatanytarsus arduenensis, V. triangularis, Nanocladius parvulus, $N$. rectinervis, etc., cada una de ellas con diferente densidad y en distinta época del año. Sólo cuatro especies aparecen como exclusivas de este grupo: Orthocladius rubicundus y Cladopelma viridula en la estación 5, Orthocladius Pe 10 en la estación 6 y Boreoheptagyia legeri en la 7, aunque dado el reducido número de ejemplares capturados, su presencia no tiene significación especial en el conjunto del río. Dentro de este grupo, la estación 4 se revela como la más independiente, desde el punto de vista cuantitativo, mientras que, cualitativamente, su composición faunística no difiere de las otras estaciones. Es muy probable que las diferencias debidas a la distinta frecuencia de aparición de las especies en esta estación, se deban a la influencia conjunta de las disminuciones de caudal provocadas por el embalse de Portodemouros y a la acción puntual de las aguas ácidas de escorrentía provenientes de la mina de cobre, situada a orillas de un afluente que desemboca inmediatamente antes de la estación, si bien no se llega a manifestar un estado de alteración persistente en lo que a la fauna de quironómidos se refiere.

\section{DISCUSIÓN}

Los datos bibliográficos referentes a la distribución longitudinal y a las preferencias ecológicas de las especies son a menudo contradictorios. De 
nuestras observaciones se desprende que muchas de las especies estudiadas se distribuyen ampliamente a lo largo del río y presentan un carácter más eurioico que el señalado en la literatura consultada.

En el río Ulla la influencia del embalse produce un estrés hidráulico importante sobre las poblaciones de quironómidos. Las variaciones de caudal a la salida de los embalses hidroeléctricos pueden ocasionar una reducción considerable de la diversidad (Fischer \& LAVOY, 1972; Trotzky \& Gregory, 1974; Gore, 1977; BroOKer \& HemsWORTH, 1978; CRISP \& RoBSON, 1979; PrAT, 1981; O'Hop \& Wallace, 1983; Canton et al., 1984). La reducción del número de especies de quironómidos se produce generalmente cuando los caudales varían de forma drástica, como han señalado GazAgnes \& Laville (1985) en el Nordeste d'Aure, lo que concuerda con lo observado por nosotros inmediatamente después del embalse de Portodemouros. Por el contrario, estos autores manifiestan que un flujo regular del embalse aumentaría el número de especies, lo que podría explicar lo observado por WARD \& STANFORD (1979) y por Chauvet (1983), quienes encuentran que las poblaciones de quironómidos se potencian a la salida de los embalses y son el grupo que mejor tolera (junto con los tricópteros filtradores, los anfípodos y los oligoquetos) las variaciones de caudal.

Algunos autores, ante la imposibilidad de efectuar muestreos distribuidos uniformemente a lo largo del año, han especulado sobre la efectividad de realizar dos o incluso un sólo muestreo en determinadas épocas del año, con la pretensión de obtener una lista de especies relativamente completa que fuese lo suficientemente representativa como para apreciar las condiciones ecológicas de un río. WILSON $(1977,1980)$, ha señalado que los períodos de emergencia máxima se sitúan en primavera y a principios del verano; precisa, además, que la época más favorable se sitúa en el mes de junio y mediados de septiembre. A este respecto Viaud-Chauvet (1982), estudiando las poblaciones de quironómidos del Aveyron (río de llanura), señala el mes de julio como la época más favorable para obtener el muestreo más representativo (78 \% de las especies). Laville (1981), estudiando el río Lot, desarrolla un programa de muestreos basado en dos series de deriva, que rea- liza durante el verano (mediados de junio y julio). Si comparamos la evolución anual de la riqueza específica, se aprecia de inmediato que los máximos valores del Ulla se registran durante la primavera y el verano. Así, la época más favorable de captura, para el conjunto de los quironómidos, sería el mes de mayo $(64,1 \%$ de las especies capturadas).

En realidad la utilización de un período de muestreo reducido ofrece una imagen muy parcial de las poblaciones de quironómidos, pues a menudo se subestima la magnitud de las variaciones estacionales de la riqueza específica de los principales grupos taxonómicos; LAVILLE (1981) señala por ejemplo, que los Diamesinae, precoces en su emergencia, estarían pobremente representados en las muestras estivales. Nosotros constatamos que este fenómeno afecta también, en gran medida, a los dos grupos más importantes de quironómidos (Orthocladiinae y Chironomini) , cuyas épocas favorables son netamente diferentes. En efecto, una muestra de julio no proporciona más que el $46 \%$ de los Orthocladiinae, frente al $100 \%$ de los Chironomini; y por el contrario, una muestra de marzo proporciona el $74,6 \%$ de los Orthocladiinae y el $57,1 \%$ de los Chironomini.

\section{BIBLIOGRAFTA}

Brooker, M. P. \& R. J. Hemsworth, 1978. The effect of the release of an artificial discharge of water on invertebrate drift in the R. Wye, Wales. Hydrobiologia 59: 155-163.

Canton, S. P., L. Cline, L. A. Shot \& J. V. WARd, 1984. The macroinvertebratesand fish of a Colorado stream during a penod of fluctuating discharge. Freshwat. Biol. 14: 311-316.

Chauvet, R., 1983. Influence d'une réduction de débit sur un torrent de montagne: l'Aston (Ariège). Annls. Limnol. 19 (1): 45-49.

Cobo, F., 1988. Los Quironómidos (Diptera, Chironomidae) de los ríos Ulla y Sar: Estudio faunístico y ecológico. Tesis doctoral, Universidad de Santiago de Compostela.

Cobo, F., M. A. González \& J. Ramil, (en prensa). Quironómidos (Diptera: Chironomidae) del río Ulla, I. Bol. Asoc. esp. Entomol.

Crisp, D. T . \& S. Robson, 1979. Some effects of discharge upon the transport of animals and peat in a northern Pennine headstream. J. appl. Ecol. 16: 721-736.

Fischer, S. G. \& A. LAvoy, 1972. Différences in littoral fauna due to fluctuating water levels below an hydroelectric dam. J. Fish. Res. Bd. Can. 29: 1472-1476. 
Gazagnes, G. \& H. Laville, 1985. Étude faunistique des Chironomidés (Diptera) de la Haute Neste d'Aure (Pyrénées Centrales): impact des aménagements hydroélectriques. Annls. Limnol. 21 (2): 149-159.

GORE, J. A., 1977. Reservoir manipulations and benthic macroinvertebrates in a prairie river. Hydrobiologia 55: 113-123.

KLEIN, L., 1959. River pollution-I: Chemical Analysis. Butterworths, London. 206 pp.

Laville, H., 1981. Récolte d'exuvies de Chironomides (Diptera) dans le Haut-Lot de la source $(1295 \mathrm{~m})$, au confluent de la Truykre (223 m). Annls. Limnol. 17 (3): 255-289.

Nisbet, M. \& J. Verneaux, 1970. Composants chimiques des eaux courantes. Discussion et proposition de classes en tant que bases d'interprétation des analyses chimiques. Annls. Limnol. 6: 161-190.

O'Hop, J. \& J. B. Wallace, 1983. Invertebrate dnft discharge, and sediment relations in a sourthern Appalachian headwater stream. Hydrobiologia 98: 71-84.

Pearson, D. L., 1977. A pantropical comparison of bird community structure on six lowland forest sites. The Condor 79: 232-244.

PRAT, N., 1981. The influence of reservoirs discharge on bentic fauna in the river Ter, N. E. Spain. Proc. 3rd int. Symp. Trichoptera. Ser. Ent. Dr. W. Junk b.v. Publishers, The Hague, Neth. 20: 293-301.
RaAbe, E. W., 1952. Uber den Affinitatswert in der Planzensoziologie. Vegetatio. Haag. 4: 53-68.

RODRIGUEZ, R., 1982. La transición morfoclimática en la cuenca del Ulla. Monograf. Univ. Santiago, 70: 230 pp.

Sokal, R. R. \& C. D. Michener, 1958. A statistical method for evaluating sistematic relationships. Univ. Kansas Sci. Bull. 38: 1409-1438.

SORENSEN, T., 1948. A method of establishing groups of equal amplitude in plant sociology based on similanty of species content and its application to analyses of the vegetation on Danish commons. Biol. Skr. 5: 1-34.

Trotzky, H. M. \& R. M. Gregory, 1974. The effect of water flow manipulation below an hydroelectnc power dam on the bottom fauna of the Upper Kennebec River, Maine. Tram. Am. Fisch. Soc. 103: 318-324.

VIAUd-Chauvet, M., 1982. Analyse des récoltes d'exuvies nymphales de Chironomides dans les rivières $d u$ bassin $d u$ Tarn. Thèse 3ème cycle. Ecologie. U.P.S. Toulouse. $171 \mathrm{pp}$.

WARD, J. V. \& J. A. STANFORD, 1979. Symposium summary and conclusions: 377-385. In: J. Ward \& J. A. Stanford (Eds.), The ecology of regulated streams. New York.

WILSON, R. S., 1977. Chironomid pupal exuviae in the River Chew. Freshwat. Biol. 7: 9-17.

WILson, R. S., 1980. Classifying nvers using Chironomid pupal exuviae. In: D. A. Murray (Ed.), Chironomidae, Ecology, Systematics, Citology and Physiology, p. 209-216. Pergamon Press. Oxford. 